\title{
Efficient Discovery of Internet Gateways in Future Vehicular Communication Systems
}

\author{
Marc Bechler, Lars Wolf \\ Technical University of Brunswick, Germany \\ Email: [bechler, wolf]@ibr.cs.tu-bs.de
}

\author{
Oliver Storz \\ Lancaster University, UK \\ Walter J. Franz \\ DaimlerChrysler AG, Germany \\ Email: o.storz@lancaster.ac.uk Email: walter.franz@daimlerchrysler.com
}

\begin{abstract}
Future road communication scenarios are expected to deploy a radio ad hoc communication technology for the transmission of data between vehicles. Such ad hoc networks are able to support low latencies and cost efficiency, which is very important for, e.g., safety related applications. In addition to vehicle-to-vehicle communication, users also will be interested in accessing Internet services from within the vehicular network. Access can be gained by using roadside installed Internet Gateways, which are able to communicate with the vehicles. However, several difficulties must be addressed in such a scenario. Examples are the interoperability of communication protocols, mobility support, communication efficiency, the discovery of Internet Gateways, and the handover of connections from one gateway to the next. In this paper, we are focussing on the aspect of discovering the Internet Gateways. We therefore developed a Service Discovery Protocol, which is highly optimized for the characteristics of future vehicular ad hoc networks. Besides the benefit of efficient service discovery, our protocol is able to choose the most suitable Internet Gateway among others using fuzzy methods. Furthermore, evaluations show that we are able to decrease the overhead caused by the service discovery process significantly.
\end{abstract}

\section{INTRODUCTION}

Future developments in the automobile domain will also include the utilization of new communication technologies. The major goals are to provide increasing automotive safety, to achieve smooth traffic flow on the roads, and to improve the convenience of vehicles' passengers by providing them with information and entertainment. In order to avoid communication costs and to be able to guarantee the low delays required for the exchange of safety related data between cars, inter-vehicle communication (IVC) systems based on ad hoc networks are a promising solution for future road communication scenarios. Vehicles thereby are able to organize themselves locally in ad hoc networks without any preinstalled infrastructure. Communication in future IVC systems will not be restricted to neighbored vehicles traveling within the radio transmission range. Like in typical ad hoc networking scenarios, the IVC system will also provide multihop communication capabilities by using "relaying" vehicles that are traveling between the sender and receiver. Fig. 1 illustrates this basic idea. In this particular example, vehicle $v 1$ is still able to communicate with vehicle $v 4$, although $v 4$ is

\footnotetext{
${ }^{0}$ The project "FleetNet - Internet on the Road" is partly funded by the German Ministry of Education and Research $(\mathrm{BMB}+\mathrm{F})$ under contract number
} $01 \mathrm{AK} 025$.

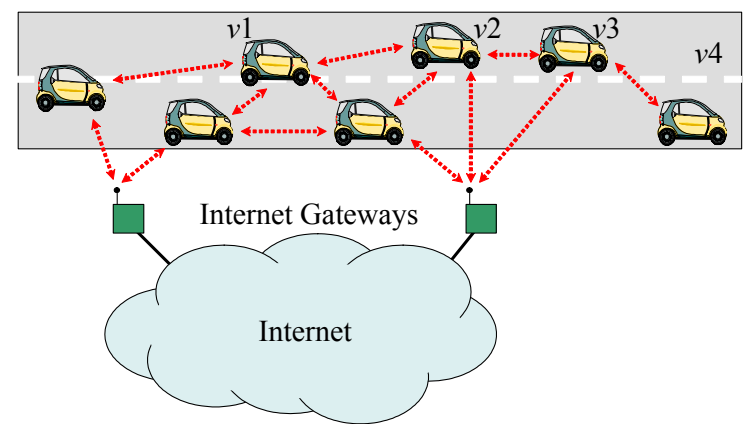

Fig. 1. Future Vehicular Communication Scenario

not in $v 1$ 's immediate communication range. Vehicle $v 2$ and $v 3$ thereby acts as an intermediate vehicle, relaying the data to the receiver. As a result, the multi-hop capabilities of the IVC system significantly increases the virtual communication range, as it enables communication with more distant vehicles.

Besides multi-hop vehicle-to-vehicle communications, future IVC systems will also comprise roadside installed gateways to the Internet. The Internet Gateways (IGWs) are on one hand integrated into the IVC system, on the other hand, they are connected to the Internet. The IGWs provide a cheap, however timely restricted access to the Internet for passing vehicles. However, those IGWs must be discovered by the vehicles in the ad hoc network. In contrast to conventional ad hoc networks formed by most other mobile devices (e.g., PDAs or laptops), vehicular ad hoc networks are highly mobile and dynamic, i.e. the network topology changes frequently. As a result, the availability of IGWs changes frequently, too, and several gateways might be available at the same time.

In this paper, we propose an efficient and scalable solution for the discovery of Internet gateways in such a highly mobile vehicular environment. Our approach deploys an intelligent fuzzy system for deciding which gateway currently fits best according to the requirements of the vehicle. In the next section, we will introduce the basic communication schemes and their Internet-working in future vehicular network. We will also discuss possible solutions to identify the IGWs. Section III will then introduce our approach in detail, followed by an evaluation in section IV. Finally, section V will conclude this paper. 


\section{COMmunicAtion In FUtURE IVC Systems}

A basic project for future inter-vehicle communication is FleetNet [1]. Its key design factors are the capability to distribute locally relevant data and to satisfy the needs of drivers and passengers of vehicles for location-dependent information and services. FleetNet therefore developed a radio protocol for ad hoc networking. Vehicles are able to communicate either directly or using intermediate vehicles as relaying nodes (multi-hop, cf. fig. 1). Hence, vehicles are able to communicate also with distant Internet Gateways using multi-hop communication. In order to access Internet resources, we need an adequate communication architecture for Internet integration, as described in the following section.

\section{A. Internet Integration of Future Vehicular Networks}

In IVC systems, communication typically takes place on a protocol layer below IP, i.e. in a sublayer of the network layer in the ISO/OSI model. This sublayer performs the multihop forwarding capabilities. In FleetNet, we deploy a positionbased routing scheme. The forwarding algorithm depends on the position of the vehicles, which is provided by a distributed location service [1]. In order to support IP-based applications, each vehicle has to be identifiable through a unique IP (version 6) address. Hence, the IVC system can be considered as one complex IPv6 subnet with various access routers (the IGWs) to the Internet. The Internet integration protocols have to bring together the different communication protocols deployed in the IVC system and the Internet. Examples are the different IP addressing schemes (version 4 vs. version 6), mobility support, and different transport layer protocols used for efficient communications. We therefore developed a proxy-based communication architecture, which handles these differences. In order to support the mobility of vehicles, we combined our architecture with a (modified) version of Mobile IP. This approach enables the communication from the Internet to the vehicles independent of their current speed and location. A detailed description can be found in [2]. The Internet Gateways thereby play an important role, as they connect the vehicular communication network physically to the Internet. The IGWs stack an IP-based layer onto the top of the IVC system to assimilate the different addressing schemes used in the IVC system and the Internet respectively. Hence, IPbased applications running within the vehicles are able to communicate with hosts in the Internet.

Due to the vehicles' mobility, they pass IGWs permanently. Gateways appear in the vehicular ad hoc network as common (but stationary) nodes. Therefore, the Internet integration requires a suitable protocol to identify the IGWs effectively. This identification must function even using multihop communication between the vehicle and the IGW. Another important issue is the scalability of such a protocol, as future IVC systems may comprise a potentially large number of vehicles.

\section{B. Related Work}

The problem of discovering IGWs in future vehicular communication systems is similar to the challenge of finding services in ad hoc networks. This identification could be achieved either at the network layer, or at the application layer.

1) Network Layer Solutions: On the network layer, two general solutions are possible. One approach is the use of multicast for the identification of IGWs. The IGWs form a (predefined) multicast group. This way, a vehicle sends its Internet traffic to this group, i.e. to all IGWs within its virtual communication range. If there are $n$ IGWs in the virtual communication range, the data will be transmitted to the Internet $n$ times. An optimization would be the use of anycast, which supports the addressing of one member of a group. Although multi-/anycast seem easy and straightforward in theory, they are hard to realize. Both require a respective support for the routing protocol deployed in the vehicular ad hoc network. Additionally, the group maintenance causes much overhead in such a highly mobile environment.

An alternative network layer approach is the modification of router discovery process. This approach is realized, e.g., in MIPMANET [3]. The basic idea is that routers advertise themselves within the local network. Hence, each mobile node is able to configure its default router according to the advertisements it receives. However, in vehicular ad hoc networks the router advertisements must be flooded in the network, because each vehicle itself acts as a router. As a result, the overall ad hoc network will be flooded periodically with router advertisements. As vehicular networks might become very large, this approach does not scale with the number of participating vehicles. Hence, this approach seems unusable for future vehicular communication scenarios, too.

2) Service Discovery Protocols: In large-scale networks, the configuration and usage of mobile devices could be very difficult. A new user has to configure her or his mobile device manually, e.g. to print a document on the nearest printer. Service discovery protocols address such problems. They enable users, mobile devices, and applications to discover services in a network with a minimum or completely without prior knowledge. Currently, various service discovery protocols are available, such as the Service Location Protocol (SLP), Universal Plug and Play (UPnP), Jini, or Salutation [4], [5]. In the following, we describe SLP representatively to point out the basic mechanisms of service discovery protocols.

Standardized in RFC 2608 [6], SLP origins from the Internet community. SLP's very basic are agents, which appear on behalf of service providers, users, or service directories. SLP differentiates three different types of agents:

- Service Agent (SA): SAs act on behalf of service providers. Their task is to propagate addresses and characteristics of the provided services.

- User Agent (UA): UAs are consulted by applications to find specified services within a network. UAs therefore communicate with SAs and Directory Agents.

- Directory Agent (DA): DAs implement a service direc- 


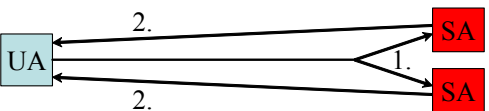

1. Service Request (SrvRqst, Multicast)

2. Service Reply (SrvRply)

(a) Service Discovery Without DAs

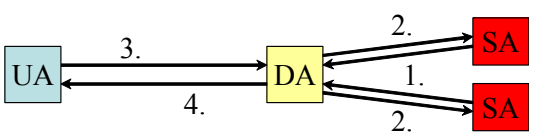

1. Service Register (SrvReg)

2. Acknowledgement (SrvAck)

3. Service Request (SrvRqst)

4. Service Reply (SrvRply)

(b) Service Discovery Using DAs

Fig. 2. Protocol Interaction in SLP

tory, which manages the available services in a network.

SLP specifies two basic interaction modes for discovering services, as illustrated in fig. 2. The first discovery process (fig. 2(a)) shows the basic types of interactions between entities in networks without DAs. Instead, each SA has to join a predefined multicast group. A UA queries available SAs by sending a service request (SrvRqst) to this multicast address. Afterwards, each SA replies to the query in the second step with a service reply (SrvRply). Note that SLP uses Service URLs (Uniform Resource Locators [6]) to specify the location of a service, which could be an IP address. The second interaction mode deploys one or more DAs, as shown in fig. 2(b). Each SA within the network has to register its service(s) with the DA using a service registration message (SrvReg, step 1). The DA acknowledges the registration with a SrvAck message (step 2). A UA requests a service immediately from a DA (SrvRqst, step 3), which replies with the registered services (SrvRply) in step 4. In this interaction mode, only the DAs need to be discovered once as illustrated in the first mode.

The classical service discovery paradigm is reactive, i.e. it is up to the mobile devices to 'initiate' the service discovery. Service requests are transmitted either immediately to a directory service, or they are transmitted to all participants in the network using multicast. This approach works well in (small to large) local area networks, because typically the number, type, and configuration of services is quite static. The mobility of devices is rather low in corporate networks, too. Hence, service requests will likely occur when a new device joins the network, or before the first usage of a service. In large-scale networks, the use of service directories further improves the scalability of service discovery process, as multicast is only required for the discovery of the service directories.

However, vehicles usually travel at relatively high speeds. As a result, their service landscape varies permanently. This characteristic particularly has effects when considering the stationary IGWs. In order to achieve a consistent view on the available IGWs, vehicles permanently have to discover new gateways. In case of a high vehicle density on a motorway, this permanent discovery heavily burdens the vehicular ad hoc network. Depending on the routing algorithm deployed, the multi-hop capability multiplies this effect. Hence, we developed DRIVE, a new protocol to discover IGWs in future vehicular networks, which is described in detail throughout the following section.

\section{DRIVE}

The service discovery of IGWs must be scalable even if the vehicles' density increases. In order to discover IGWs efficiently, we developed and implemented DRIVE (DiscoveRy of Internet gateways from VEhicles). Its implementation bases on SLP described previously. Besides scalability and efficiency, another important object is to determine the most suitable IGW among the available gateways. Hence, the service discovery process of DRIVE comprises two functional tasks: the discovery of available IGWs, and the selection of the most suitable IGW.

\section{A. Discovery of Available Internet Gateways}

The key concept of DRIVE is the commutation of the roles of service user and service provider. This way, IGWs do not wait for requests from vehicles requiring Internet access. Instead, they themselves advertise their service proactively. Vehicles looking for a service assume a passive role, i.e. they do not discover the gateways actively. Hence, we call this process "passive discovery" further on.

In order to enable passive discovery in DRIVE, we divided the functionality of a service provider into two distributed units (cf. fig. 3). The first unit is situated on the IGWs and announces their service periodically. The service advertisements are transmitted using the geocast capabilities of the vehicular communication system. If a vehicle moves into the (virtual) transmission range of an IGW, the second functional unit within the vehicle will receive the gateway's service advertisements. It then extracts the service information and stores it in a local database. Due to the periodicity of the service advertisements, a vehicle is able to infer whether an IGW is available or not. From vehicle's point of view, the discovery of IGWs is reduced to a search in the vehicle's local database. If the search is successful, the in-vehicle functional unit will respond with the respective IGW. Otherwise, the user must assume that a gateway is currently not available.

The benefits of DRIVE are obvious. DRIVE prevents the copious transmission of service requests (cf. section IV). Another very important benefit is that no multicasting capabilities are needed within the vehicular ad hoc network. The traffic caused by the service advertisements in DRIVE is based upon geocast only. In contrast, traditional service discovery protocols like SLP require a respective multicast support of the ad hoc routing algorithm. Moreover, the network traffic caused by DRIVE is correlated with the location of 


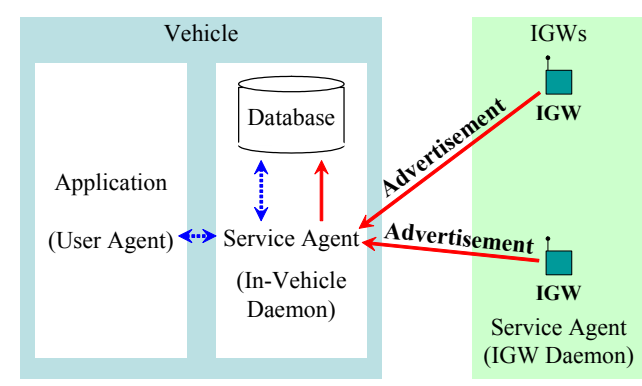

Fig. 3. Service Discovery in DRIVE

the IGWs. In traditional service discovery protocols, vehicles permanently have to proactively scan their environment for (newly) available IGWs. Hence, the discovery process of each vehicle permanently sends service request packets, which causes overhead even if no gateway is available.

\section{B. Selection of Suitable Internet Gateways}

Due to the multi-hop nature of the vehicle network, several IGWs might be within a vehicle's communication range at the same time. A selection has to be made by the DRIVE communication architecture, deciding which IGW to use for connecting to the Internet. The outcome of this selection process will significantly influence the quality of service experienced while using the selected gateway for accessing the Internet. For example, choosing an IGW that that resides within direct communication range of a vehicle's wireless networking hardware will help to keep the communication delay between IGW and vehicle at a very low rate. However, it is very likely that a new Internet Gateway has to be discovered after driving out of the direct coverage area of the IGW, leaving the vehicle with a potential period of disconnection during the handoff process. In order to be able to make an optimized selection, the decision process should be based on the requesting application's expectations regarding the quality of service. A simple classification scheme helps DRIVE to estimate these expectations. Each application can specify its requirements using a combination of the following properties: Interactivity, Streaming and Real-Time. Besides the application's requirements, DRIVE's selection mechanism also takes into account various types of state information about the vehicle network and the available IGWs. The vehicle network can, for example, provide data about the current traffic density in the vicinity of a vehicle. However, the largest amount of state information is carried in piggy-back manner within the IGWs' periodical service advertisements, including information about

- the current number of clients using the current IGW,

- the utilisation of the IGW's available bandwidth,

- the IGW's geographical position for the estimation of the connection's duration and the communication delay, and

- Additional marketing information, allowing the IGW operators to send local advertisements to the vehicles.

Note that this collection of possible parameters is not complete. Further parameters might also be relevant for the

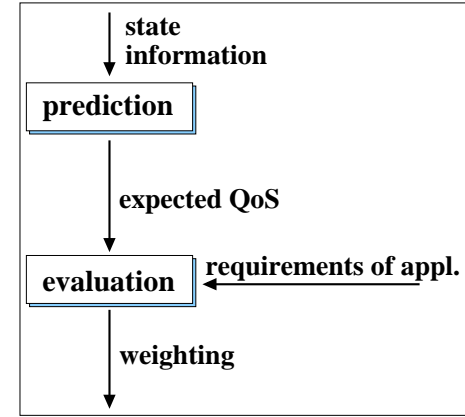

Fig. 4. DRIVE's two-step selection process

selection process, depending on the nature of future applications deployed in this scenario. The integration of those parameters is rather simple by adding new fields into the service announcements. Having acquired the necessary information, DRIVE uses a fuzzy-logic approach for carrying out the decision process. Fig. 4 shows an outline of DRIVE's twostep approach. In the first phase, a fuzzy engine predicts the expected quality of service for a particular IGW. Currently, we use the following input parameters for our predictions: current traffic density, distance to the IGW, current number of users, and the current bandwidth. Based upon this information, the fuzzy engine calculates the following expected parameters of the next IGW:

- expected disconnection probability

- expected number of users for the next IGW

- expected bandwidth utilization of the next IGW

- expected packet loss probability

Of course, it is nearly impossible to formalize typical traffic scenarios as the vehicles' movements depend, among other factors, on human drivers. Predictions are therefore only possible at a high level of abstraction. Hence, fuzzy logic seems to be a very promising approach as it allows the formulation of the coherences on such a high degree of abstraction. The second phase of DRIVE's decision process uses another fuzzy engine to evaluate the predicted quality of service by means of the application's requirements. Hence, the fuzzy engine calculates an IGW's performance for each application class, i.e. interactive, streaming, and real-time. The result is a weighted order of available IGWs, ordered according to their individual suitability for the needs of the requesting application.

\section{EVAluation}

The evaluation focuses on a mathematical analysis of DRIVE and SLP. We concentrate our evaluation on the worstcase scenario. The overhead analysis of a service discovery protocol thereby depends on many factors. One important factor is the performance of the routing protocol deployed in the vehicular ad hoc network. The worst-case scenario assumes the use of local flooding (with duplicate detection) for the transmission of a message. Furthermore, we assumed the following parameters for our investigations: 
- $l_{\text {geo }}=12 \mathrm{~km}$ (IGW's virtual communication range)

- $r=1 \mathrm{~km}$ (vehicle's radio transmission radius)

- $n_{\text {lane }}=6$ lanes (number of lanes, both directions)

- $P_{a d v}=P_{\text {rqst }}=110$ byte (average size of advertisement/service request)

In order to determine the maximum overhead (using flooding with dupliate detection), we have to derive the maximum traffic caused by SLP and DRIVE first. The use of SLP in a vehicular ad hoc network requires the following (estimated) bandwidth $B_{S L P}$ in the worst case [2]:

$$
\begin{aligned}
& B_{S L P}=\# \text { SLP SrvRqsts } * \text { message size } \\
& \leq 2 \cdot r \cdot\left(\rho \cdot n_{\text {lanes }}\right)^{2} \cdot l_{\text {geo }} \cdot f_{\text {rqst }} \cdot P_{\text {rqst }}
\end{aligned}
$$

$\rho$ denotes the traffic density, $f_{\text {rqst }}$ the frequency of the service requests transmitted by a vehicle. Similarly, the maximum bandwidth requirements $B_{D R I V E}$ of DRIVE can be estimated by the following obvious inequality [2]:

$$
\begin{aligned}
B_{D R I V E} & =\text { \# DRIVE advertisements } * \text { message size } \\
& \leq 2 \cdot r \cdot \rho \cdot n_{\text {lanes }} \cdot\left(\frac{l_{\text {geo }}}{2 r}+1\right) \cdot f_{a d v} \cdot P_{a d v}
\end{aligned}
$$

with the traffic density $\rho$ and the advertisement periodicity $f_{a d v}$. As we see in this worst-case scenario, the (dynamical) traffic density occurs quadratically in SLP, whereas it is a linear factor in DRIVE. Fig. 5 visualizes the dependency between the bandwidth requirements and traffic density for both SLP and DRIVE. The difference between the two curves in the diagram is the frequency for the transmission of the service requests and service advertisements. We chose frequencies of 1 request/advertisement and 0.2 request/advertisement per second. Note the different scales on the axis of ordinates. As can be seen in fig. 5, DRIVE outperforms SLP. For example, if the traffic density is 20 vehicles per kilometer and lane, the use of SLP will cause an overhead of approximately 60 Mbps. In contrast, DRIVE is content with about 300 kbps (both with 1 request/advertisement per second). Note that the estimation of $B_{S L P}$ considers the service requests only. Usually, the IGWs have to respond to each request, which multiplies the bandwidth requirements in the worst-case scenario accordingly.

\section{CONClusion}

Access to Internet resources in future road communication scenarios will be very important for the passengers' convenience. This access will be possible using stationary Internet Gateways at the roadside, which open up the Internet for vehicular networks. However, those gateways must be discovered by the passing vehicles. In this paper, we described possible solutions for the realization of such a discovery process. We showed that neither network layer approaches nor the use of classical service discovery protocols are able to fulfill the requirements of the targeted scenario. Hence, we proposed DRIVE, an efficient and scalable protocol for discovering Internet Gateways in future road communication scenarios. One key concept of DRIVE is the deployment of announcements,

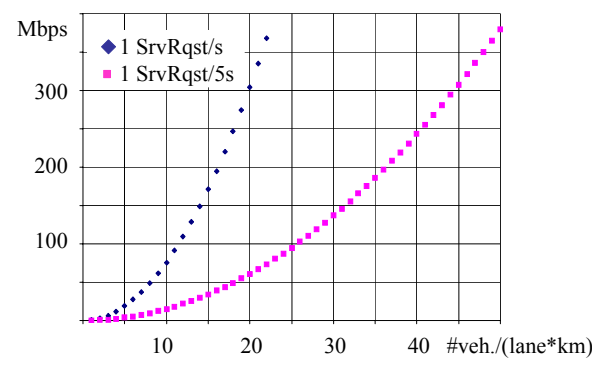

(a) SLP

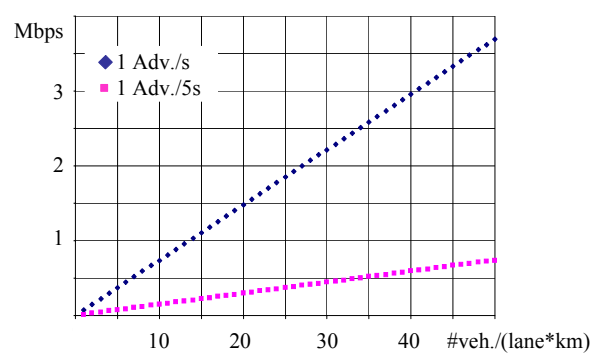

(b) DRIVE

Fig. 5. Comparison: SLP vs. DRIVE

which advertise the presence of the Internet Gateways. Due to this proactive nature, DRIVE scales well compared to classical service discovery protocols. The overhead caused by DRIVE basically depends on the number of Internet Gateways, which is expected to be very small compared to the number of vehicles participating in communication activities.

Another very important feature of DRIVE is the automatical selection of the most suitable Internet Gateway for communications. In future road communication scenarios, several Internet Gateways could be available at the same time. They could be accessed either directly, or via other intermediate vehicles driving in front or behind the vehicle. DRIVE therefore uses a fuzzy-approach based on various network characteristics and application classes. Hence, DRIVE is able to significantly improve communication efficiency by choosing the gateway that currently fits best to the application's requirements.

\section{REFERENCES}

[1] W. Franz et. al., FleetNet - Internet on the Road, Proceedings of 8th World Congress on Intelligent Transportation Services, Sydney, Australia, Oct. 2001.

[2] M. Bechler et. al., Mobile Internet Access in FleetNet, 13. Fachtagung Kommunikation in verteilten Systemen, Leipzig, Germany, Apr. 2003.

[3] U. Jörnsson et. al, MIPMANET - Mobile IP for Mobile Ad Hoc Networks, Proc. ACM International Symposium on Mobile Ad Hoc Networking and Computing, Boston, USA, Aug. 2000.

[4] C. Lee and S. Helal, Protocols for Service Discovery in Dynamic Mobile Networks, Int. Journal of Computer Research, Special Issue on Wireless Systems and Mobile Computing, 2002.

[5] G. G. Richard III, Service Advertisement and Discovery: Enabling Universal Device Cooperation, IEEE Internet Computing, Sep./Oct. 2000.

[6] E. Guttman et al., Service Location Protocol, Version 2, Request for Comment 2608, Internet Engineering Task Force, Jun. 1999. 